This article is not an exact copy of the original published article in Methods of Information in Medicine (ISSN: 0026-1270). The definitive publisher-authenticated version of:

R. Sassi, M. W. Rivolta, L. T. Mainardi, R. C. Reis, M. O. C. Rocha, A. L. P. Ribeiro and F. Lombardi, Spatial Repolarization Heterogeneity and Survival in Chagas Disease, Methods of Information in Medicine, vol. 53(6), pp. 464-468, 2014. DOI: 10.3414/ME14-01-0002.

is available online at: http://dx.doi.org/10.3414/ME14-01-0002.

\title{
Spatial repolarization heterogeneity and survival in Chagas disese
}

R. Sassi ${ }^{1}$, M. W. Rivolta ${ }^{1}$, L. T. Mainardi' ${ }^{2}$, R. C. Reis ${ }^{3}$, M. O. C. Rocha ${ }^{3}$, A. L. P. Ribeiro ${ }^{3}$, F. Lombardi $^{4}$

${ }^{1}$ Dipartimento di Informatica, Università degli Studi di Milano, Italy;

${ }^{2}$ Dipartimento di Elettronica, Informazione e Bioingegneria, Politecnico di Milano, Italy;

${ }^{3}$ Faculdade de Medicina e Hospital das Clínicas, Universidade Federal de Minas Gerais, Brazil;

${ }^{4}$ Cardiologia, Fondazione IRCCS "Policlinico di Milano", Dipartimento di Scienze Cliniche e di Comunità, Università degli Studi di Milano, Italy.

Address of the corresponding author:

Roberto Sassi

Dipartimento di Informatica, Università degli Studi di Milano

Via Bramante 65, 26013 Crema (CR), Italy

Phone/FAX: +3902 503 30056/+3902503 30074

E-mail: roberto.sassi@unimi.it 


\section{Summary}

Objectives: We investigate if cardiac spatial repolarization heterogeneity might be associated with an increased risk of death in patients with chronic Chagas disease.

Methods: Repolarization heterogeneity was assessed using the $V$-index, a recently introduced metric founded on a biophysical model of the ECG. This metric provides an estimate of the standard deviation of the repolarization times across the heart. We analyzed 113 patients (aged 21-67 years) enrolled between 1998 and 1999 who had a known serological status showing a positive reactions to Trypanosoma cruzi. 14 subjects died during a 10 year followup period.

Results: The $V$-index was significantly lower in survivor (S) than in non-survivor (NS) subjects (S: $31.2 \pm 13.3$ ms vs NS: $41.2 \pm 18.6$ ms, single-tail t-test: $p=0.009$, single-tail Wilcoxon rank sum test: $p=0.029)$. A $V$-index larger than 36.3 ms was related to a significantly higher risk of death in a univariate Cox proportional-hazards analysis (hazard ratio, HR $=5.34, p=0.0046$ ). In addition, $V$-index $>36.3$ ms retained its prognostic value in a multivariate Cox proportionalhazards analysis after adjustment for other 3 clinical variables (left ventricular ejection factor $<0.50$, QRS duration $>133 \mathrm{~ms}$, ventricular tachycardia during stress testing or 24 hours Holter) and for T-wave amplitude variability $>30 \mu \mathrm{V}$, even using shrinkage, a statistical procedure that protects against over-fitting due to small sample size.

Conclusions: The study showed that an increased dispersion of repolarization times in patients with by Chagas disease, as measured by the $V$-index, is significantly correlated with the risk of death in a univariate survival analysis. The $V$-index captures prognostic information not immediately available from the analysis of other established risk factors.

Keywords: Chagas Disease [C03.752.300.900.200], Chagas Cardiomyopathy [C03.752.300.900.200.190], Ambulatory Electrocardiography [E01.370.370.380.240.230], Digital Signal Processing [L01.224.800], Electrophysiological Processes [G07.265.337], Ventricular Fibrillation [C14.280.067.922] 


\section{Introduction}

American trypanosomiasis, also called Chagas disease, is a parasitic infection widely present in central and South America. Due to migration of infected persons from endemic areas to developed countries, Chagas disease patients now can be found also in Europe and the United States. Carriers may stay asymptomatic for decades. It is induced by Trypanosoma cruzi, a protozoa that elects as its preferential sites of multiplication the myocardial and nervous fibers. As a consequence, in the chronic phase of the disease, it frequently causes a progressive deterioration of the heart [1]. Chagas disease is still one of the main causes of sudden death in Latin America [2].

In particular, chronic Chagas disease (cChD) might affect the anatomy of the myocardium (myocarditis, cardiomegaly), the electrophysiology of the heart and the autonomic innervations. Hence, sinus block (SB), the atrioventricular block (AVB), inter-ventricular block (RBBB and $\mathrm{LBBB}$ ) and premature ventricular contraction (PVC) are typically observed. The autonomic neuropathy that characterizes most patients with Chagas disease may also contribute to the development of heart failure or the occurrence of syncope, bradyarrhythmia or tachyarrhythmia [1-4]. Different prognostic parameters have been utilized in the last 15 years, to improve death risk stratification and enabling specific therapy [5-8]. Until now, however, risk stratification in Chagas disease patients remains an open issue.

In the present study, we made the assumption that as a result of an abnormal enlargement of the myocardium and structural changes of cardiac myocytes, cChD might also alter the spatial distribution of the myocytes' repolarization times across the heart. In support of our hypothesis, it must be recalled that: i) spatial repolarization heterogeneity creates suitable conditions for the mechanism of reentry and favors the development of ventricular fibrillation [9], commonly observed in cChD; ii) previous studies have found an association between QT variability [10] or T-wave amplitude variability [11], and sudden cardiac death; iii) and, more recently, we have reported [8] that an increase in T-wave amplitude variability (TWV) was associated with an increased risk of death in cChD.

We therefore analyzed the same ECG recordings utilized in the previous study [8] but quantified spatial repolarization heterogeneity with the $V$-index, a metric recently introduced [12]. Based on a biophysical model, this parameter is an estimate of the standard deviation of the repolarization times across the heart. We therefore tested whether patients' mortality could be predicted by a structural remodeling of the repolarization times, as detected by the $V$-index, a parameter capable of reflecting spatial repolarization heterogeneity due to the dispersion of the myocytes' repolarization times across the ventricles.

\section{Methods}

\subsection{Dataset}

The study was performed on data collected at the Chagas Disease Outpatient Reference Center of the Universidade Federal de Minas Gerais, Brazil and was designed as an observational cohort study. The data were previously described in $[7,8]$.

Briefly, 113 patients aged 21-67 (mean: 42.5) were enrolled between 1998 and 1999 and completed a 10-year follow-up period. The patients had a definite serological status showing a positive reaction to Trypanosoma cruzi. Many laboratory analysis and ambulatory tests were collected at enrolment, among which a 10 minutes Holter recording, at rest in controlled 
conditions. The recordings were performed with a Burdick (Altair) digital Holter recorder with $1000 \mathrm{~Hz}$ sampling frequency and 10- or 16-bit resolution.

Patients were followed until death or the end of the follow up in 2008. Death cases were not categorized, so no distinction was made between sudden, cardiac and noncardiac death. Out of 113 subjects, 14 died during a 10 year follow-up period.

\subsection{Assessing repolarization heterogeneity}

About a decade ago, van Oosterom [13], using a biophysical model showed that the shape of the T-wave on surface ECG in one single beat, $\Psi(t)$, (a vector with a row for each of the $L$ leads), can be related to the myocytes' transmembrane potentials during repolarization, via the approximate relation

$$
\boldsymbol{\Psi}(t) \approx \underbrace{-\mathbf{A} \Delta \boldsymbol{\rho}}_{\boldsymbol{w}_{1}} T_{d}(t)+\underbrace{1 / 2 \mathbf{A} \Delta \boldsymbol{\rho}^{2}}_{\boldsymbol{w}_{2}} \frac{d T_{d}(t)}{d t}
$$

The heart is subdivided in a certain number $M$ of sources or "nodes". Given the fact that, in first approximation, the decay of the transmembrane potential during repolarization is very similar across myocytes, a single function $T_{d}(t)$ takes the place of its first derivative in the previous equation. What changes across nodes is the repolarization time $\rho_{m}$. Hence $\bar{\rho}=$ $\frac{1}{M} \sum_{m=1}^{M} \rho_{m}$ is the average repolarization time in a beat and, most importantly, $\Delta \rho_{m}=\rho_{m}-$ $\bar{\rho}$ is the repolarization delay of the node $m$ with respect to the entire set. In equation (1), $\Delta \boldsymbol{\rho}=\left[\Delta \rho_{1}, \ldots, \Delta \rho_{M}\right]^{T}$ is a vector of repolarization delays and $\Delta \boldsymbol{\rho}^{2}=\left[\Delta \rho_{1}^{2}, \ldots, \Delta \rho_{M}^{2}\right]^{T} . \mathbf{A}$ is a patient-dependent $[L \times M]$ transfer matrix accounting for the contribution of each node to the $L$-leads electrocardiographic recording in $\boldsymbol{\Psi}(t)$. The terms $\boldsymbol{w}_{1}$ and $\boldsymbol{w}_{2}$ are [ $\left.L \mathrm{x} 1\right]$ vector of lead factors, one for each lead.

Sassi \& Mainardi recently [12] introduced a statistical model for the repolarization delay $\Delta \rho_{m}$ for each node $m$ over a short interval of time where the heart rate (HR) does not vary significantly:

$$
\Delta \rho_{m}(k)=\vartheta_{m}+\varphi(k)
$$

where $\vartheta_{m}$ models the spatial variability of the repolarization times for a given subject at a given $\mathrm{HR}$, and $\varphi_{m}(k)$ describes differences in repolarization times which are observable among successive beats, being $k$ the beat index. Please refer to the original paper for a complete discussion of the validity of the model. Here we briefly summarize the main assumptions: i) each source in the heart has a constant-in-time repolarization delay $\vartheta_{m}$ (with respect to $\bar{\rho}$ ); ii) for each node, fluctuations of repolarization times across following beats are modeled as a normal random variable, i.e. $\varphi(k) \sim N\left(0, \sigma_{\varphi}^{2}\right)$; iii) the random oscillations have the same intensity $\sigma_{\varphi}$ in each source. Under these assumptions, it is possible to prove that

$$
s_{\vartheta}=\left(\sum_{m=1}^{M} \frac{\vartheta_{m}^{2}}{M}\right)^{1 / 2} \approx \frac{\operatorname{std}\left[w_{2}(i)\right]}{\operatorname{std}\left[w_{1}(i)\right]}=V_{\mathrm{i}}
$$

where the standard deviations (std) are computed on the lead factors of lead $i$ across a certain number of consecutive beats (not across different leads). The term $V_{i} \approx s_{\vartheta}$, the $V$-index, is an estimate of the standard deviation of the repolarization times, across the myocardium. In particular of that part, $\vartheta_{m}$, which does not vary in the period of time under analysis. 


\subsection{Computational details}

The $V$-index was estimated for each subject in the dataset. First, each record was processed for QRS detection and classification using a refined version of the freely available software OSEA [14]. The ECG signals were preliminarily band-pass filtered in the range 0.5$40 \mathrm{~Hz}$ to remove major baseline wander and high-frequency disturbances ( $3^{\text {rd }}$ order Butterworth filter, forward and reverse application to avoid phase distortions). To reduce the impact of misclassification of the labeling routine, an average template was built using the normal beats and then used to mark as abnormal those ones which had a correlation factor with the template smaller than 0.9.

Then the detection of a few fiducial points was performed on the template. In details, the $J$ point was roughly selected as the first minimum after the $\mathrm{R}$ peak on the vector magnitude (VM) signal (the square root of the sum of the three orthogonal leads squared). As for the VM signal the end of the T-wave was determined according to Surawicz's method, i.e. the point at which the tangent of the maximum slope intersects the baseline [15]. It must be noticed that the computation of the $V$-index does not require an accurate detection of such fiducial markers but they were used to bracket, roughly, the position of the T-wave on the ECG. The template was also employed to reset the baseline level to zero [12], after being offset by the filtering procedure.

It has been known for long time that the heart rate modulates the duration of the QT interval. Also, its duration is different during adaptation to a different heart rate [16]. Given the link between spatial repolarization heterogeneity and QT duration, the model (2) better performs when the heart rate is stationary. The ECG recordings were collected at rest in controlled conditions, so abrupt changes in HR were unlikely. However, we selected for the subsequent analysis only those beats which: i) were "stable" under the definition of equation (1) in [16]; ii) had a preceding NN interval which was close enough to the mean of the population. Such range was selected as small as possible, but large enough to contain at least 60 beats (for a direct comparison with [8]). On average, we considered only beats with NN intervals differing up to \pm 15 ms from the mean.

Finally, the lead factors were computed using the algorithms described in the appendix of [12] and the index in equation (3) evaluated. As we had $L=3$ estimates for each subject, we considered their mean.

\subsection{Statistical analysis}

The survival statistical analysis was performed using the Survival package in the R software [17].

TWV and three traditional clinical risk factors were shown to be independent predictors of death in $\mathrm{CChD}$, in a previous study on the same population [8]. Hence, we further tested the hypothesis that the $V$-index might be an independent prognostic factor, in a multivariate models including: prolonged QRS duration (>133 ms), left ventricular ejection fraction (LVEF, measured using the Simpson's method) below 50\%, ventricular tachycardia at either Holter monitoring or stress testing and Median TWV $>30 \mu \mathrm{V}$. The determination of these factors was described in details in [8] while the computational details for TWV are in [11].

The multivariate survival analysis was performed using the R software, as well. Given the relatively small sample size, modeling strategies were enforced to avoid the risk of overfitting. The "optimism" of the model (to be limited) was evaluated according to Harrell [18], using the RMS package ("validate"). Adjusted ("shrunk") regression coefficients were calculated using the linear shrinkage method [19]. The shrinkage factor was given by $\mathrm{s}=$ 
$\frac{\left(\chi^{2}-\mathrm{df}\right)}{\chi^{2}}$, where $\chi^{2}$ and $\mathrm{df}$ are the likelood ratio and the number of degrees of freedom of the model, respectively.

\section{Results}

The computation of the $V$-index was performed on each recording. For three cases it led to a value larger than $100 \mathrm{~ms}$, which was due to poor quality of the signals and/or small number of stable beats. They were excluded from further analysis. The rest of the population had a $V$-index of $39.4 \pm 12.8 \mathrm{~ms}$. The computation included an average of $76 \pm 19$ beats (not necessarily adjacent).

When we compared the values obtained from patients who died in the follow-up period (NS) in comparison to survivors (S), the average values showed a difference (S: $31.2 \pm 13.3 \mathrm{~ms}$ vs NS: $41.2 \pm 18.6 \mathrm{~ms}$ ). This difference was statistically significant (single-tail t-test: $p=0.009$, single-tail Wilcoxon rank sum test: $p=0.029$ ). A box plot of the metrics is given in Fig. 1 ; it should be noted that several outliers are present in the survivor group.

The $V$-index was dichotomized at the cut value $36.3 \mathrm{~ms}$, which was the one which maximized positive and negative predictability in the ROC curve (the point at which sensitivity and specificity were equivalent and equal to $71.4 \%$ ). The area under the curve was small (0.672, but this is not surprising as in the study the observational window was, by design, limited in time).

A $V$-index larger than 36.3 ms was related to an elevated risk of death in a univariate Cox proportional hazards analysis (hazard ratio, $H R=5.34, p=0.0046$ ). However, when not dichotomized, the $V$-index did not prove to be clearly related to an elevated risk of death ( $p$ $=0.14)$. Kaplan-Meier curves are reported in Fig. 2 . The log-rank chi square test $(p=0.0022)$ suggests a significant distinction on mortality across the two populations.

Finally, once dichotomized at $36.3 \mathrm{~ms}$, the $V$-index retained its prognostic value in a Cox proportional-hazards analysis, after adjustment for the other four variables considered. The significance was preserved for any of the five factors, even when using the shrinkage method that protects against over-fitting due to small sample size. Table 1 contains the results of the analysis. The shrinkage factor was $s=0.918$ (likelihood ratio $\chi^{2}=56.71$, degrees of freedom: 5 ).

\section{Conclusions}

The main finding of our study was that dispersion of repolarization times, as measured by the $V$-index, was significantly correlated with the risk of death in a univariate survival analysis. In particular, a value of $V$-index larger than $36.3 \mathrm{~ms}$ selected a population of cChD patients with a higher risk of death $(p=0.0022)$. The study employed recordings where only 3 leads were available. While the leads redundancy exploited was smaller than in [12], the present work suggested that the $V$-index can be effectively estimated in such situations. Indeed, numerical simulations meant to investigate the accuracy-loss, i.e. quantified on synthetic ECG recordings, confirmed this observation [20].

The work was performed after the study of Ribeiro et al. [8], which showed that repolarization variability, as measured by T-wave amplitude variability (TWV), was related to the risk of death in Chagas disease. In this work instead, spatial heterogeneity of repolarization was linked to an increase risk of death in cChD. It is important to recall that these two methodologies are based on different measures and if anything they could be considered as complementary. In fact, whereas TWV mainly reflects beat to beat variability 
in myocytes' repolarization times (quantified in the model by $\sigma_{\varphi}$ ), $V$-index reflects their spatial variability within the same cardiac cycle and is considered to be constant across consecutive beats (quantified in the model by $s_{\vartheta}$ ). In fact, in first approximation, using the mathematical formalism of section 2.2, the variance of the amplitude of the T-wave (at any instant $t$ in lead $i)$ is [21]:

$$
\operatorname{var}\left[\Psi_{i}(t)\right]=\sigma_{\varphi}^{2} T_{d}^{2}(t) \sum_{m=1}^{M} A_{i, m}^{2}
$$

Using the categorization given in [22], the $V$-index is a "static" repolarization assessment while TWV a "dynamic" one. Our findings suggest that both mechanisms may be relevant to unmask alteration in repolarization patterns as those present in patients with Chagas disease. The multivariate analysis further supported this statement. Both TWV and $V$-index contemporarily proved predictors of mortality, independently of other clinical parameters such as LVEF and QRS length. Hence, these results supported the conclusion that both temporal and spatial repolarization heterogeneities are modified significantly by the profound changes in the anatomy and electrophysiology of the myocardium, induced by cChD.

As a final remark, it must be noticed that the dispersion of the myocytes' repolarization times measured by the $V$-index should not be confused with "QT dispersion", which is a measure of the variability of QT interval duration among different leads within the same cardiac cycle. Although QT dispersion was proposed as a measure of spatial dispersion of ventricular recovery times [23], it was limited by the inaccuracy of T-wave end determination thus leading over the years to inconsistent results and major criticism [22].

The study presents several limitations. The sample size is relatively small, especially when considering the number of deaths; however, this limitation was partially overcome by the use of the shrinkage method. The mode of death was not precisely determined in this cohort. However, sudden death is the most common cause of death in cChD patients [2]: indeed, in one of the largest cChD cohorts, $62.3 \%$ of patients died of sudden death [5]. Thus, sudden death is probably the main cause of death in this sample too. Finally, we did not compare the standard deviation of $\boldsymbol{w}_{1}$ and $\boldsymbol{w}_{2}$ (being both parameters largely dependent on $\sigma_{\varphi}$ ) with TWV or micro T-wave alternans.

In conclusion, we showed that an increased dispersion of repolarization times in patients with Chagas disease, as measured by the $V$-index, is significantly correlated with the risk of death in both univariate and multivariate survival analysis. The clinical utility of this new noninvasive technique in identifying patients at risk of sudden cardiac death should be evaluated in further studies.

\section{REFERENCES}

1. Laranja FS, Dias E, Nobrega G, Miranda A. Chagas' Disease: A Clinical, Epidemiologic, and Pathologic Study. Circulation. 1956;14(6):1035-60.

2. Rassi A Jr, Rassi SG, Rassi A. Sudden death in Chagas' disease. Arq Bras Cardiol. 2001;76(1):8696.

3. Amorim DS, Godoy RA, Manço JC, Tanaka A, Gallo L. Effects of Acute Elevation in Blood Pressure and of Atropine on Heart Rate in Chagas' Disease: A Preliminary Report. Circulation. 1968;38(2):289-94. 
4. Nunes MCP, Dones W, Morillo CA, Encina JJ, Ribeiro AL. Chagas Disease: An Overview of Clinical and Epidemiological Aspects. J Am Coll Cardiol. 2013;62(9):767-76.

5. Rassi A Jr, Rassi A, Little WC, Xavier SS, Rassi SG, Rassi AG, et al. Development and Validation of a Risk Score for Predicting Death in Chagas' Heart Disease. N Engl J Med. 2006;355(8):799-808.

6. Rassi A Jr, Rassi A, Rassi SG. Predictors of Mortality in Chronic Chagas Disease A Systematic Review of Observational Studies. Circulation. 2007;115(9):1101-8.

7. Ribeiro ALP, Cavalvanti PS, Lombardi F, Nunes MDCP, Barros MVL, Rocha MODC. Prognostic Value of Signal-Averaged Electrocardiogram in Chagas Disease. J Cardiovasc Electrophysiol. 2008;19(5):502-9.

8. Ribeiro ALP, Rocha MODC, Terranova P, Cesarano M, Nunes MDCP, Lombardi F. T-Wave Amplitude Variability and the Risk of Death in Chagas Disease. J Cardiovasc Electrophysiol. 2011;22(7):799-805.

9. Kuo CS, Munakata K, Reddy CP, Surawicz B. Characteristics and possible mechanism of ventricular arrhythmia dependent on the dispersion of action potential durations. Circulation. 1983;67(6):1356-67.

10. Haigney MC, Zareba W, Gentlesk PJ, Goldstein RE, Illovsky M, McNitt S, et al. QT interval variability and spontaneous ventricular tachycardia or fibrillation in the Multicenter Automatic Defibrillator Implantation Trial (MADIT) II patients. J Am Coll Cardiol. 2004;44(7):1481-7.

11. Couderc J-P, Zareba W, McNitt S, Maison-Blanche P, Moss AJ. Repolarization variability in the risk stratification of MADIT II patients. Europace. 2007;9(9):717-23.

12. Sassi R, Mainardi LT. An Estimate of the Dispersion of Repolarization Times Based on a Biophysical Model of the ECG. IEEE Trans Biomed Eng. 2011;58(12):3396-405.

13. Van Oosterom A. Genesis of the T wave as based on an equivalent surface source model. J Electrocardiol. 2001;34(4, Part B):217-27.

14. Hamilton P. Open Source ECG Analysis software (OSEA). www.eplimited.com; 2003.

15. Lepeschkin E, Surawicz B. The Measurement of the Q-T Interval of the Electrocardiogram. Circulation. 1952;6(3):378-88.

16. Badilini F, Maison-Blanche P, Childers R, Coumel P. QT interval analysis on ambulatory electrocardiogram recordings: a selective beat averaging approach. Med Biol Eng Comput. 1999;37(1):71-9.

17. Therneau T. A Package for Survival Analysis in S. R package version 2.36-12. 2012.

18. Harrell FE. Regression Modeling Strategies: With Applications to Linear Models, Logistic Regression, and Survival Analysis. Springer; 2001.

19. Steyerberg EW. Clinical prediction models: a practical approach to development, validation, and updating. New York: Springer; 2009.

20. Sassi R, Mainardi LT. Quantification of spatial repolarization heterogeneity: Testing the robustness of a new technique. Computing in Cardiology (CinC), 2012. 2012. pag. 69-72.

21. Mainardi LT, Sassi R. Some theoretical results on the observability of repolarization heterogeneity on surface ECG. J Electrocardiol. 2013;46(3):270-5.

22. Malik M, Batchvarov VN. Measurement, interpretation and clinical potential of QT dispersion. J Am Coll Cardiol. 2000;36(6):1749-66.

23. Day CP, McComb JM, Campbell RW. QT dispersion: an indication of arrhythmia risk in patients with long QT intervals. Br Heart J. 1990;63(6):342-4. 


\section{Figure Legends}

Fig. 1. Box plot of the $V$-index values, distinguishing patients who were known to have died (NS) before the end of study and those who instead survived (S).

Fig. 2. Kaplan-Meier survival curves built using the $V$-index as a stratifier (dichotomized at the value $36.3 \mathrm{~ms}$ ). The $p$ values corresponding to the log-rank statistics of the Kaplan-Meier survival models was $p=0.0022$. 
Figure 1

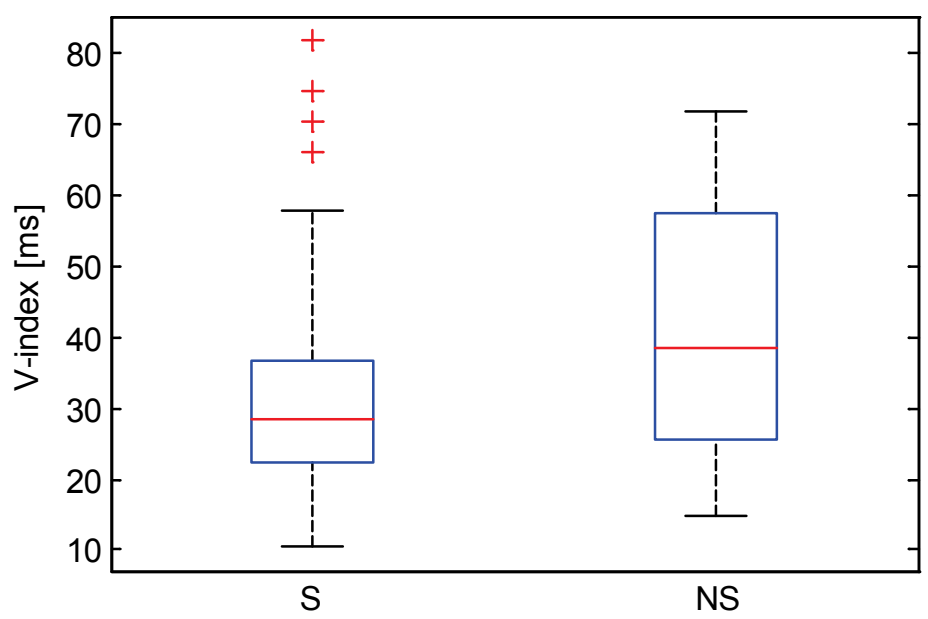


Figure 2

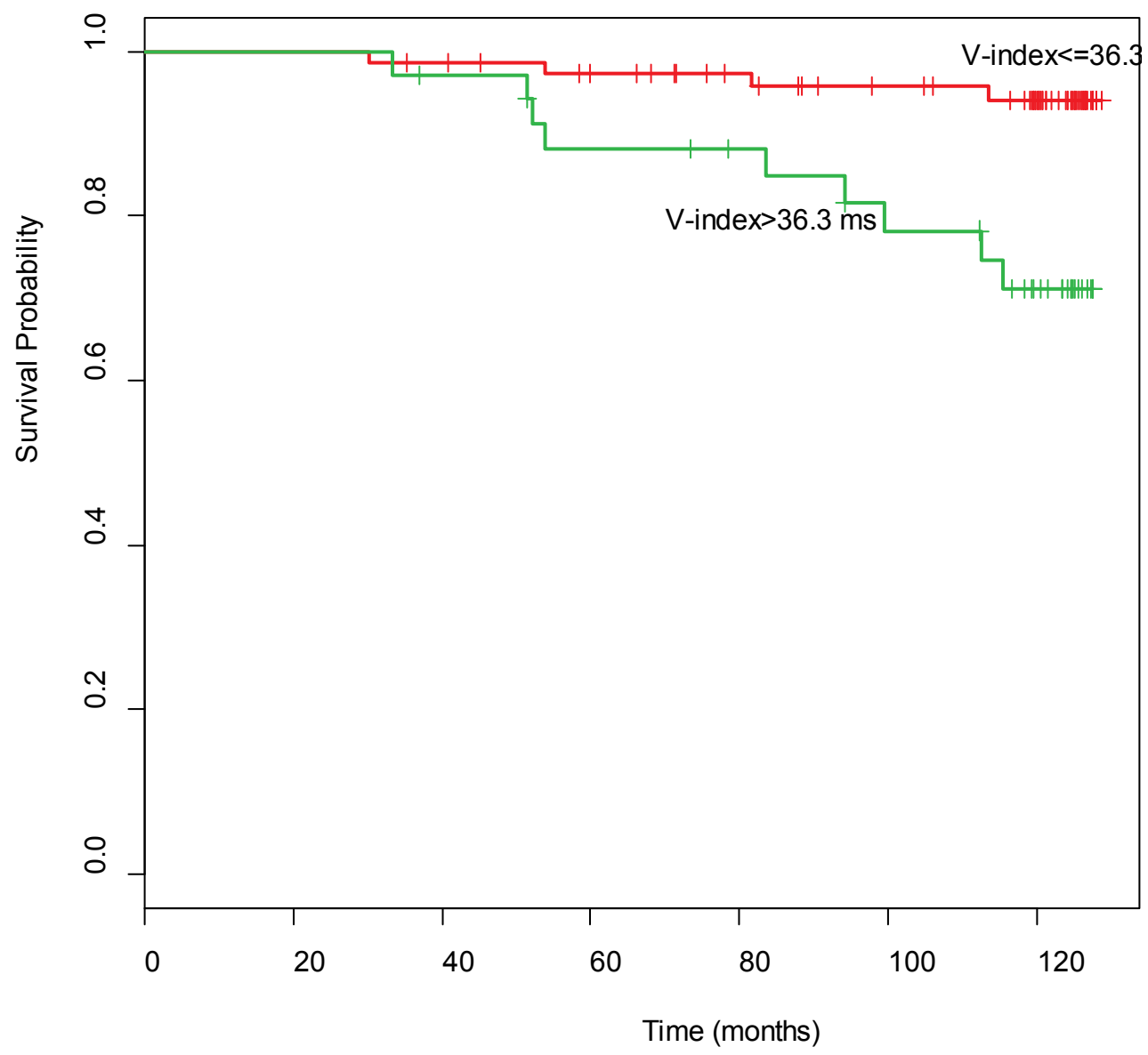


Table 1. Multivariate Cox Proportional-Hazards Analysis of risk of death in Chagas disease patients

\begin{tabular}{|c|c|c|c|c|c|}
\hline & ß coefficient & $\begin{array}{l}\text { Shrunk } \\
\text { ß coefficient }\end{array}$ & SE & $\begin{array}{l}\text { Hazard } \\
\text { ratio }\end{array}$ & $95 \% \mathrm{Cl}$ \\
\hline LVEF $<50 \%$ & 1.80 & 1.65 & 0.71 & 5.19 & $1.30-20.73$ \\
\hline $\begin{array}{l}\text { VT at stress testing } \\
\text { or } 24 \mathrm{~h} \text { Holter }\end{array}$ & 2.24 & 2.05 & 0.82 & 7.74 & $1.56-38.38$ \\
\hline QRS > $133 \mathrm{~ms}$ & 2.14 & 1.95 & 0.82 & 7.05 & $1.42-35.01$ \\
\hline Median TWV $>30 \mu V$ & 2.44 & 2.22 & 0.85 & 9.24 & $1.75-48.66$ \\
\hline $\mathrm{V}$ index $>36.3 \mathrm{~ms}$ & 1.52 & 1.39 & 0.66 & 4.00 & $1.09-14.66$ \\
\hline
\end{tabular}

LVEF = left ventricular ejection fraction; VT = ventricular tachycardia; Median TWV = median value of T-wave amplitude variability. Hazard ratio calculated according to the shrunk $\beta$ coefficients. 